\title{
Mannich-Type Reaction of Aldimines with 2-Silyloxydienes Catalyzed by Ammonium Chloride
}

\author{
Shoichi Fukumoto, Miho Shigenobu, Kaori Ishimaru \\ Department of Applied Chemistry, National Defence Academy, Hashirimizu, Yokosuka, Japan \\ Email: kaoriisi@nda.ac.jp
}

How to cite this paper: Fukumoto, S., Shigenobu, M. and Ishimaru, K. (2019) Mannich-Type Reaction of Aldimines with 2-Silyloxydienes Catalyzed by Ammonium Chloride. International Journal of Organic Chemistry, 9, 163-173.

https://doi.org/10.4236/ijoc.2019.94014

Received: September 19, 2019

Accepted: November 23, 2019

Published: November 26, 2019

Copyright $\odot 2019$ by author(s) and Scientific Research Publishing Inc. This work is licensed under the Creative Commons Attribution International License (CC BY 4.0).

http://creativecommons.org/licenses/by/4.0/

\begin{abstract}
Reaction of imines with 2-silyloxydiene catalyzed by ammonium chloride has been perfectly proceeded under environmentally friendly conditions to give Mannich-type product selectively. The reaction would proceed via Mannichtype mechanism, not cyclization/ring-opening process. Cyclopropanation of the corresponding Mannich-type product gave the precursor of prasugrel skeleton in high yield.
\end{abstract}

\section{Keywords}

Mannich-Type Reaction, Ammonium Chloride, Imine, 2-Silyloxydiene

\section{Introduction}

Mannich-type reactions are widely recognized as a powerful method for constructing a variety of b-aminoketones [1]-[6]. However, Mannich-type reaction of imines with 2 -silyloxydienes, which provides easy access to $\beta$-aminoketones having a terminal olefin, is still challenging because $[4+2]$ type cycloadducts [7]-[15] or mixtures of Mannich-type products and cycloadducts [16] [17] [18] are obtained in most cases, as shown in Figure 1. Previously we first reported a highly effective Mannich-type reaction of imine with 2-silyloxydiene in the presence of zinc triflate and water [19] [20] [21] [22], which gave the corresponding $\beta$ '-amino- $\alpha, \beta$-enones as attractive skeletons for pharmaceutically useful compounds [23] [24] [25] [26]. Although many vinylogous Mannich-type reactions have been developed [27]-[36], only a few examples that describe the selective preparation of $\beta^{\prime}$-amino- $\alpha, \beta$-enones by the reaction of imine with 2 -silyloxybutadiene have been reported so far. Thus, Kawęcki isolated the open-chain products from the aza-Diels-Alder reaction of sulfinimines with the 


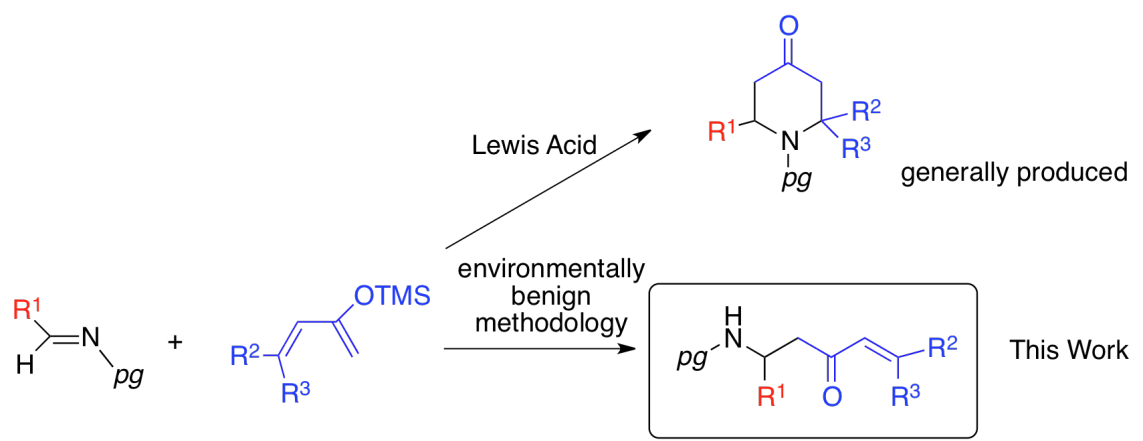

Figure 1. Reaction of imine with 2-silyloxydiene.

Rawal diene [37]. Pan et al. reported the addition of an $\alpha, \beta$-unsaturated ketonederived enolate to chiral $N$-phosphonyl imines [38], and Prasad et al. developed the reaction of chiral sulfinimines with silyloxydiene using TMSOTf [39]. In spite of these recent achievements, a more economical and environmentally benign synthetic methodology using green and sustainable catalysts has not been reported yet that offer alternatives to metal catalysts. Here we report the ammonium chloride-catalyzed Mannich-type reaction of imines with 2-silyloxybutadienes under mild conditions.

\section{Results and Discussion}

Initially, we examined the reaction of imine 1a, derived from benzaldehyde and $o$-anisidine, with 2-silyloxybutadiene 2 a (Table 1 ). In contrast to the similar aza-Diels-Alder reaction of electron-rich Danishefsky's diene, reported by Ding et al., which afforded the cyclic product in $\mathrm{MeOH}$ in the absence of any acids [40], the reaction of imine 1a with 2-silyloxybutadiene 2a in EtOH or $\mathrm{MeOH}$ without additives gave no product (Table 1, entry 1). This result suggested that

Table 1. Mannich-type reacton of imine (1a) with 2-silyloxydiene (2a) ${ }^{\mathrm{a}}$.

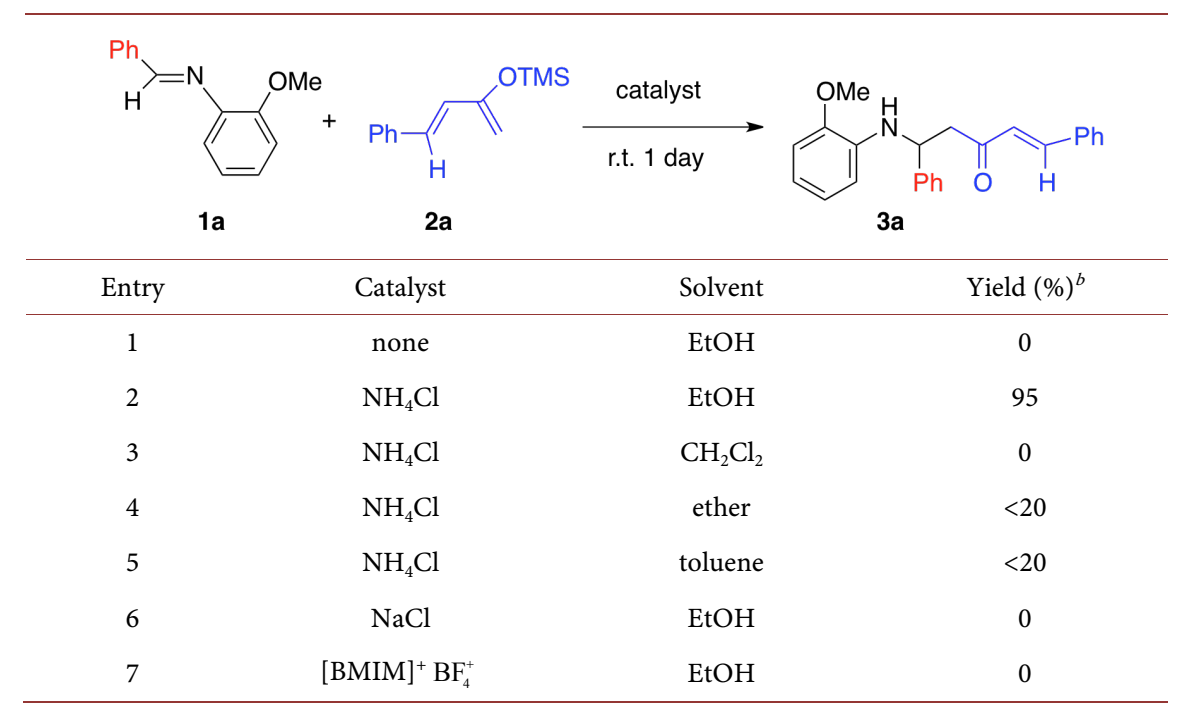

${ }^{\mathrm{a}}$ Conditions: imine 1 ( $\left.1 \mathrm{mmol}\right), 2$-silyloxydiene $2(1.2 \mathrm{mmol})$, catalyst $(0.1 \mathrm{mmol})$ in dry solvent $(1 \mathrm{~mL})$, r.t., 1 day. ${ }^{\text {b } I s o l a t e d ~ y i e l d s . ~}$ 
using additive or catalyst was necessary to promote the reaction. We found that reaction with ammonium chloride $(10 \mathrm{~mol} \%)$ as a catalyst in $\mathrm{EtOH}$ gave the corresponding Mannich-type product 3a selectively in 95\% isolated yield (entry 2). Interestingly, no trace of cycloadduct was detected by $500 \mathrm{MHz}{ }^{1} \mathrm{H}$ NMR spectroscopy in the crude product. The previously reported reaction of imines having the $N$-benzyl group [19] did not give any products using ammonium chloride in EtOH, indicating that the reactivity of the imine is largely dependent on the $N$-protecting group. Finally, the attempt to perform the reaction using other additives and solvents was unsuccessful (Table 1, entries 3 - 7).

Having established the optimal reaction conditions for the Mannich-type reaction, we subsequently explored the scope of the reaction with respect to the imine substrates (Table 2). Imines $1 \mathrm{~b}$ and $1 \mathrm{c}$ bearing an $o$ - or $p$-tolyl group reacted to provide the corresponding products $3 \mathbf{b}$ ( $90 \%$ yield) and $3 \mathbf{c}$ ( $88 \%$ yield), respectively. Meanwhile, imines having an electron-donating or an electron-withdrawing group all reacted in a satisfactory way to provide the corresponding products $\mathbf{3} \mathbf{d}-\mathbf{3} \mathbf{g}$ in high yield. The reaction with 2 -silyloxybutadiene $\mathbf{2 b}$, derived from mesityl oxide, also proceeded to give $3 \mathbf{h}-3 \mathbf{k}$ in $87 \%-97 \%$ yields. Further investigation of the reaction with 2 -silyloxydiene $2 \mathrm{c}$ derived from acetylcyclohexene afforded the corresponding $\beta^{\prime}$-amino- $\alpha, \beta$-enones in $95 \%-98 \%$ yields (Table 3).

To investigate the reaction mechanism, the reaction of $1 \mathrm{a}$ and $2 \mathrm{a}$ was quenched after $1 \mathrm{~h}$ and analyzed using $500 \mathrm{MHz}{ }^{1} \mathrm{H}$ NMR spectroscopy. No cycloadduct was detected but a Mannich-type product and starting materials were observed. We suspect that the Mannich-type products are not formed via cyclization/ring-opening mechanism, as in the case of our previous reaction between $N$-benzyl-protected imine and 2-silyloxybutadiene using zinc triflate and water (Scheme 1) [19]. Additionally, further $\mathrm{HCl}$ work-up of the acyclic product 3a gave no cycloadducts but the $\beta^{\prime}$-amino- $\alpha, \beta$-enone was recovered. However, $N$-benzyl-protected acyclic products afforded piperidones upon reaction with $\mathrm{HCl}$ [19], indicating that the acyclic product $\mathbf{3 a}$ is stable under acidic conditions.

To demonstrate the synthetic utility of the Mannich-type products, we performed the preparation of a precursor of the prasugrel skeleton [41] [42], as
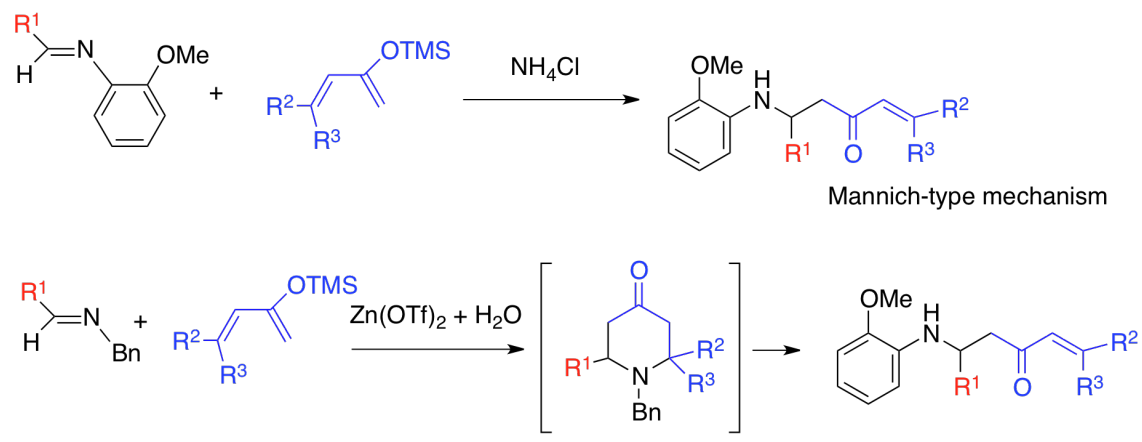

cyclization/ring-opening mechanism

Scheme 1. Plausible mechanism. 
Table 2. Scope of imines (1) and 2-silyloxydienes (2a)

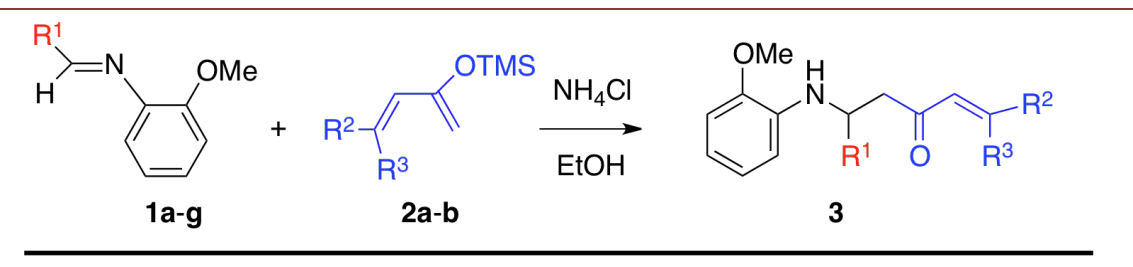<smiles>COc1ccccc1NC(CC(=O)/C=C/c1ccccc1)c1ccccc1</smiles>

3a, $95 \%$ b<smiles>COc1ccccc1NC(CC(=O)/C=C/c1ccccc1)c1ccccc1</smiles>

$3 c, 88 \%$ b<smiles>COc1ccccc1NC(CC(=O)/C=C/c1ccccc1)c1ccc(Cl)cc1</smiles>

3e, $93 \%$ b<smiles>COc1ccccc1NC(CC(=O)/C=C/c1ccccc1)c1ccc(Br)cc1</smiles><smiles>COc1ccccc1NC(CC(=O)C=C(C)C)c1ccc(C)cc1</smiles><smiles>COc1ccccc1N</smiles><smiles>COc1ccccc1NC(CC(=O)/C=C/c1ccccc1)c1ccc(C)cc1</smiles><smiles>COc1ccc(C(CC(=O)/C=C/c2ccccc2)Nc2ccccc2OC)cc1</smiles><smiles>COc1ccccc1NC(CC(=O)/C=C/c1ccccc1)c1ccccc1</smiles>

3f, $81 \%^{\mathrm{b}}$<smiles>COc1ccccc1NC(CC(=O)C=C(C)C)c1ccccc1</smiles>

3 h, $95 \%$ b<smiles>COc1ccc(C(CC(=O)C=C(C)C)Nc2ccccc2OC)cc1</smiles>
$(1 \mathrm{~mL})$, r.t., 1 day. ${ }^{\mathrm{b}}$ Isolated yields.

shown in Scheme 2. Thus, the reaction of the imine (1h) derived from $o$-fluorobenzaldehyde with 2 -silyloxybutadiene 2 b proceeded smoothly to afford 
Table 3. Reaction of imines (1) with 2-silyloxydiene (2c) derived from acetylcyclohexene $e^{\mathrm{a}}$.

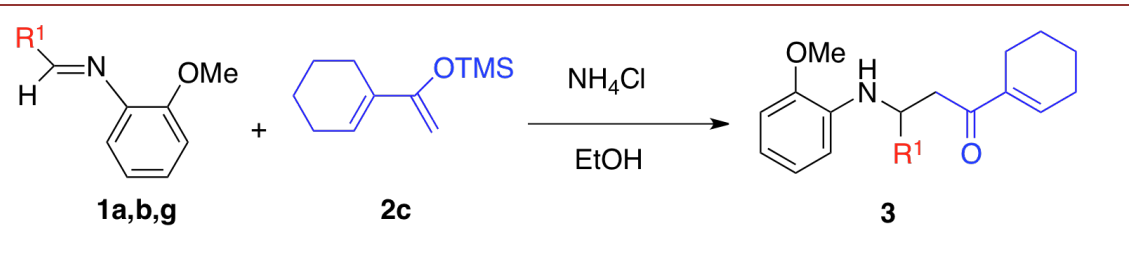<smiles>COc1ccccc1NC(CC(=O)C1=CCCCC1)c1ccccc1</smiles>

$31,98 \%$ b<smiles>COc1ccccc1NC(CC(=O)C1=CCCCC1)c1ccc(C)cc1</smiles>

$3 \mathrm{~m}, 96 \%$<smiles>COc1ccccc1NC(CC(=O)C1=CCCCC1)c1ccc(F)cc1</smiles>

3n, $95 \%$ b

${ }^{a}$ Conditions: imine $1(1 \mathrm{mmol}), 2$-silyloxydiene $2 \mathrm{c}(1.2 \mathrm{mmol})$, ammonium chloride $(0.1 \mathrm{mmol})$ in dry EtOH $(1 \mathrm{~mL})$, r.t., 1 day. ${ }^{\text {b } I s o l a t e d ~ y i e l d s . ~}$

the corresponding acyclic product 3o, which was then cyclopropanated to give 40 in $65 \%$ yield.

\section{Conclusion}

In summary, a Mannich-type reaction of imine with 2-silyloxybutadiene that provides access to versatile $\beta$-amino- $\alpha, \beta$-enones has been developed under green conditions. We found that the use of ammonium chloride in $\mathrm{EtOH}$ is critical for the efficient outcome of the reaction, and this non-metal method is useful for various dienes and aldimines compared to reports so far [37] [38] [39]. According to our results, the reaction proceeds via Mannich-type mechanism, instead of through a cyclization/ring-opening process. Additionally, we prepared a precursor of the prasugrel skeleton by cyclopropanation of 3o. Further investigation of the applicability of this reaction and mechanistic elucidation is currently in progress.

\section{Experimental}

\section{Typical Procedure for Mannich-Type reaction of imine 1 with}

\section{2-silyloxydiene 2}

To a stirred solution of $\mathrm{NH}_{4} \mathrm{Cl}(0.006 \mathrm{~g}, 0.1 \mathrm{mmol})$, imine $1(1 \mathrm{mmol})$ in dry ethanol $(1 \mathrm{~mL})$ was added 2-silyloxydiene $2(1.2 \mathrm{mmol})$ at r.t.. The reaction mixture was stirred at r.t. for $24 \mathrm{~h}$, the solvent was evaporated at reduced pressure. 
The crude product was purified by flash column chromatography (ethyl acetate: $n$-hexane $=1: 6)$ to afford 3.

Data for 3a; colorless oil, $0.34 \mathrm{~g}, 95 \% .{ }^{1} \mathrm{H}$ NMR $\left(\mathrm{CDCl}_{3}, 500 \mathrm{MHz}\right) \delta 3.17$ (dd, $1 \mathrm{H}, J=15.4 \mathrm{~Hz}, 5.7 \mathrm{~Hz}$ ), $3.23(\mathrm{dd}, 1 \mathrm{H}, J=15.4 \mathrm{~Hz}, 7.5 \mathrm{~Hz}), 3.85(\mathrm{~s}, 3 \mathrm{H}), 4.97$ $(\mathrm{dd}, 1 \mathrm{H}, J=6.9 \mathrm{~Hz}, 6.9 \mathrm{~Hz}), 6.44(\mathrm{~d}, 1 \mathrm{H}, J=7.5 \mathrm{~Hz}), 6.45-6.76(\mathrm{~m}, 4 \mathrm{H}), 7.22$ $7.25(\mathrm{~m}, 1 \mathrm{H}), 7.32-7.49(\mathrm{~m}, 10 \mathrm{H})$; HRMS (EI) $\mathrm{m} / z[\mathrm{M}]^{+}$calcd. for $\mathrm{C}_{24} \mathrm{H}_{23} \mathrm{NO}_{2}$ 357.1729, found: 357.1727 .

Data for 3b; colorless oil. $0.30 \mathrm{~g}$, 90\%. ${ }^{1} \mathrm{H}$ NMR $\left(\mathrm{CDCl}_{3}, 300 \mathrm{MHz}\right) \delta 2.02(\mathrm{~s}$, $3 \mathrm{H}), 2.87(\mathrm{dd}, 1 \mathrm{H}, J=15.4 \mathrm{~Hz}, 5.9 \mathrm{~Hz}), 2.95(\mathrm{dd}, 1 \mathrm{H}, J=15.4 \mathrm{~Hz}, 6.9 \mathrm{~Hz}), 3.54$ (s, 3H), $4.69(\mathrm{dd}, 1 \mathrm{H}, J=6.6 \mathrm{~Hz}, 6.6 \mathrm{~Hz}), 6.23(\mathrm{~d}, 1 \mathrm{H}, J=6.9 \mathrm{~Hz}), 6.35-6.51(\mathrm{~m}$, $4 \mathrm{H}), 6.78-6.87(\mathrm{~m}, 2 \mathrm{H}), 7.04-7.24(\mathrm{~m}, 8 \mathrm{H})$; HRMS (EI) $\mathrm{m} / z[\mathrm{M}]^{+}$calcd. for $\mathrm{C}_{25} \mathrm{H}_{25} \mathrm{NO}_{2} 371.1885$, found: 371.1894 .

Data for 3c; colorless oil. $0.32 \mathrm{~g}, 88 \% .{ }^{1} \mathrm{H}$ NMR $\left(\mathrm{CDCl}_{3}, 300 \mathrm{MHz}\right) \delta 2.06(\mathrm{~s}$, $3 \mathrm{H}), 2.92(\mathrm{dd}, 1 \mathrm{H}, J=15.4 \mathrm{~Hz}, 5.9 \mathrm{~Hz}), 2.96(\mathrm{dd}, 1 \mathrm{H}, J=15.4 \mathrm{~Hz}, 7.0 \mathrm{~Hz}), 3.58$ (s, 3H), $4.67(\mathrm{dd}, 1 \mathrm{H}, J=6.3 \mathrm{~Hz}, 6.3 \mathrm{~Hz}), 6.23(\mathrm{~d}, 1 \mathrm{H}, J=7.7 \mathrm{~Hz}), 6.35-6.51(\mathrm{~m}$, $3 \mathrm{H}), 6.78-6.85(\mathrm{~m}, 1 \mathrm{H}), 6.87$ - $7.07(\mathrm{~m}, 4 \mathrm{H}), 7.08$ - $7.18(\mathrm{~m}, 2 \mathrm{H}), 7.20$ - $7.26(\mathrm{~m}$, $3 \mathrm{H}$ ); HRMS (EI) $\mathrm{m} / z$ [M] $]^{+}$calcd. for $\mathrm{C}_{25} \mathrm{H}_{25} \mathrm{NO}_{2} 371.1885$, found: 371.1902 .

Data for 3d; colorless oil. $0.32 \mathrm{~g}, 85 \% .{ }^{1} \mathrm{H} \mathrm{NMR}\left(\mathrm{CDCl}_{3}, 500 \mathrm{MHz}\right) \delta 3.12$ (dd, $1 \mathrm{H}, J=15.5 \mathrm{~Hz}, 5.7 \mathrm{~Hz}$ ), $3.20(\mathrm{dd}, 1 \mathrm{H}, J=15.5 \mathrm{~Hz}, 6.9 \mathrm{~Hz}), 3.74(\mathrm{~s}, 3 \mathrm{H}), 3.83$ (s, $3 \mathrm{H}), 4.91(\mathrm{dd}, 1 \mathrm{H}, J=6.3 \mathrm{~Hz}, 6.3 \mathrm{~Hz}), 6.46(\mathrm{~d}, 1 \mathrm{H}, J=1.7 \mathrm{~Hz}), 6.61-6.63(\mathrm{~m}$, $4 \mathrm{H}), 6.81$ - $6.85(\mathrm{~m}, 2 \mathrm{H}), 7.25$ - $7.37(\mathrm{~m}, 5 \mathrm{H}), 7.42$ - 7.47 (m, 3H); HRMS (EI) $\mathrm{m} / z[\mathrm{M}]^{+}$calcd. for $\mathrm{C}_{25} \mathrm{H}_{25} \mathrm{NO}_{3} 387.1834$, found: 387.1838 .

Data for 3e; colorless oil. $0.36 \mathrm{~g}, 93 \% .{ }^{1} \mathrm{H} \mathrm{NMR}\left(\mathrm{CDCl}_{3}, 300 \mathrm{MHz}\right) \delta 3.12$ (dd, $1 \mathrm{H}, J=15.7 \mathrm{~Hz}, 5.5 \mathrm{~Hz}), 3.20(\mathrm{dd}, 1 \mathrm{H}, J=15.7 \mathrm{~Hz}, 5.5 \mathrm{~Hz}), 3.85(\mathrm{~s}, 3 \mathrm{H}), 4.93-$ $4.99(\mathrm{~m}, 2 \mathrm{H}), 6.36(\mathrm{~d}, 1 \mathrm{H}, J=6.2 \mathrm{~Hz}), 6.61-6.76(\mathrm{~m}, 4 \mathrm{H}), 7.26-7.53(\mathrm{~m}, 10 \mathrm{H})$; HRMS (EI) $\mathrm{m} / z$ [M] ${ }^{+}$calcd. for $\mathrm{C}_{24} \mathrm{H}_{22} \mathrm{NO}_{2} \mathrm{Cl} 391.1339$, found: 391.1328 .

Data for 3f; colorless oil. $0.32 \mathrm{~g}, 81 \% .{ }^{1} \mathrm{H} \mathrm{NMR}\left(\mathrm{CDCl}_{3}, 500 \mathrm{MHz}\right) \delta 3.02$ (dd, $1 \mathrm{H}, J=14.9 \mathrm{~Hz}, 8.6 \mathrm{~Hz}), 3.28(\mathrm{~d}, 1 \mathrm{H}, J=3.5 \mathrm{~Hz}), 3.71(\mathrm{~s}, 3 \mathrm{H}), 5.28(\mathrm{dd}, 1 \mathrm{H}, J=$ $9.1 \mathrm{~Hz}, 3.4 \mathrm{~Hz}), 6.21(\mathrm{dd}, 1 \mathrm{H}, J=6.2 \mathrm{~Hz}, 1.8 \mathrm{~Hz}), 6.59$ - $6.74(\mathrm{~m}, 4 \mathrm{H}), 7.17$ - 7.59 (m, 10H); HRMS (EI) $\mathrm{m} / z$ [M] $]^{+}$calcd. for $\mathrm{C}_{24} \mathrm{H}_{22} \mathrm{NO}_{2} \mathrm{Cl} 391.1339$, found: 391.1335 .

Data for 3g; colorless oil. $0.33 \mathrm{~g}, 87 \% .{ }^{1} \mathrm{H} \mathrm{NMR}\left(\mathrm{CDCl}_{3}, 500 \mathrm{MHz}\right) \delta 3.13(\mathrm{dd}$, $1 \mathrm{H}, J=15.5 \mathrm{~Hz}, 5.7 \mathrm{~Hz}$ ), $3.20(\mathrm{dd}, 1 \mathrm{H}, J=15.5 \mathrm{~Hz}, 5.7 \mathrm{~Hz}), 3.83(\mathrm{~s}, 3 \mathrm{H}), 4.93$ $(\mathrm{dd}, 1 \mathrm{H}, J=6.3 \mathrm{~Hz}, 6.3 \mathrm{~Hz}), 6.35-6.39(\mathrm{~m}, 1 \mathrm{H}), 6.59-6.80(\mathrm{~m}, 4 \mathrm{H}), 6.95$ - 6.99 (m, 2H), $7.18-7.25(\mathrm{~m}, 3 \mathrm{H}), 7.33-7.38(\mathrm{~m}, 3 \mathrm{H}), 7.45-7.53(\mathrm{~m}, 2 \mathrm{H})$; HRMS (EI) $\mathrm{m} / z[\mathrm{M}]^{+}$calcd. for $\mathrm{C}_{24} \mathrm{H}_{22} \mathrm{NO}_{2} 375.1634$, found: 375.1628 .

Data for $3 \mathrm{~h}$; colorless oil. $0.29 \mathrm{~g}, 95 \% .{ }^{1} \mathrm{H}$ NMR $\left(\mathrm{CDCl}_{3}, 300 \mathrm{MHz}\right) \delta 1.72(\mathrm{~s}$, $3 \mathrm{H}), 2.00(\mathrm{~s}, 3 \mathrm{H}), 2.76(\mathrm{dd}, 1 \mathrm{H}, J=16.9 \mathrm{~Hz}, 5.9 \mathrm{~Hz}), 2.82(\mathrm{dd}, 1 \mathrm{H}, J=16.9 \mathrm{~Hz}$, $5.9 \mathrm{~Hz}$ ), $3.81(\mathrm{~s}, 3 \mathrm{H}), 4.77(\mathrm{dd}, 1 \mathrm{H}, J=6.6 \mathrm{~Hz}, 6.6 \mathrm{~Hz}), 5.91$ (brs, $1 \mathrm{H}), 6.30-6.35$ $(\mathrm{m}, 1 \mathrm{H}), 6.45-6.55(\mathrm{~m}, 1 \mathrm{H}), 6.58-6.64(\mathrm{~m}, 2 \mathrm{H}), 7.06-7.15(\mathrm{~m}, 1 \mathrm{H}), 7.17-7.20$ $(\mathrm{m}, 2 \mathrm{H}), 7.26-7.28(\mathrm{~m}, 2 \mathrm{H})$; HRMS (EI) $\mathrm{m} / z[\mathrm{M}]^{+}$calcd. for $\mathrm{C}_{20} \mathrm{H}_{23} \mathrm{NO}_{2}$ 309.1729, found: 309.1722 .

Data for 3i; colorless oil. $0.31 \mathrm{~g}, 97 \% .{ }^{1} \mathrm{H}$ NMR $\left(\mathrm{CDCl}_{3}, 500 \mathrm{MHz}\right) \delta 1.85(\mathrm{~s}$, $3 \mathrm{H}), 2.11(\mathrm{~s}, 3 \mathrm{H}), 2.30(\mathrm{~s}, 3 \mathrm{H}), 2.90(\mathrm{dd}, 1 \mathrm{H}, J=13.2 \mathrm{~Hz}, 5.7 \mathrm{~Hz}), 2.93(\mathrm{dd}, 1 \mathrm{H}, J$ 
$=13.2 \mathrm{~Hz}, 5.7 \mathrm{~Hz}), 3.85(\mathrm{~s}, 3 \mathrm{H}), 4.85(\mathrm{dd}, 1 \mathrm{H}, J=6.9 \mathrm{~Hz}, 6.9 \mathrm{~Hz}), 6.04(\mathrm{brs}, 1 \mathrm{H})$, $6.44-6.50(\mathrm{~m}, 1 \mathrm{H}), 6.58-6.52(\mathrm{~m}, 1 \mathrm{H}), 6.55-6.73(\mathrm{~m}, 2 \mathrm{H}), 7.10-7.13(\mathrm{~m}, 2 \mathrm{H})$, 7.20 - $7.28(\mathrm{~m}, 2 \mathrm{H})$; HRMS (EI) $\mathrm{m} / z[\mathrm{M}]^{+}$calcd. for $\mathrm{C}_{21} \mathrm{H}_{24} \mathrm{NO}_{2} 323.1885$, found: 323.1894.

Data for 3j; colorless oil. $0.31 \mathrm{~g}, 92 \% .{ }^{1} \mathrm{H} \mathrm{NMR}\left(\mathrm{CDCl}_{3}, 300 \mathrm{MHz}\right) \delta 1.80$ (s, $3 \mathrm{H}), 2.08(\mathrm{~s}, 3 \mathrm{H}), 2.80(\mathrm{dd}, 1 \mathrm{H}, J=15.4 \mathrm{~Hz}, 6.2 \mathrm{~Hz}), 2.90(\mathrm{dd}, 1 \mathrm{H}, J=15.4 \mathrm{~Hz}$, $6.2 \mathrm{~Hz}), 3.70(\mathrm{~s}, 3 \mathrm{H}), 3.80(\mathrm{~s}, 3 \mathrm{H}), 4.81(\mathrm{dd}, 1 \mathrm{H}, J=6.6 \mathrm{~Hz}, 6.6 \mathrm{~Hz}), 5.95-6.00$ $(\mathrm{m}, 1 \mathrm{H}), 6.34-6.44(\mathrm{~m}, 1 \mathrm{H}), 6.52-6.58(\mathrm{~m}, 1 \mathrm{H}), 6.67-6.72(\mathrm{~m}, 2 \mathrm{H}), 6.79-6.84$ $(\mathrm{m}, 2 \mathrm{H}), 7.24-7.28(\mathrm{~m}, 2 \mathrm{H})$; HRMS (EI) $\mathrm{m} / z[\mathrm{M}]^{+}$calcd. for $\mathrm{C}_{21} \mathrm{H}_{25} \mathrm{NO}_{3}$ 339.1834, found: 339.1836 .

Data for 3k; colorless oil. 0.29 g, 89\%. ${ }^{1} \mathrm{H}$ NMR $\left(\mathrm{CDCl}_{3}, 300 \mathrm{MHz}\right) \delta 1.85$ (s, $3 \mathrm{H}), 2.10(\mathrm{~s}, 3 \mathrm{H}), 2.88(\mathrm{dd}, 1 \mathrm{H}, J=15.4 \mathrm{~Hz}, 5.9 \mathrm{~Hz}), 2.92(\mathrm{dd}, 1 \mathrm{H}, J=15.4 \mathrm{~Hz}$, $5.9 \mathrm{~Hz}), 3.11(\mathrm{~s}, 3 \mathrm{H}), 4.84(\mathrm{dd}, 1 \mathrm{H}, J=6.6 \mathrm{~Hz}, 6.6 \mathrm{~Hz}), 5.99-6.01(\mathrm{~m}, 1 \mathrm{H}), 6.36$ $(\mathrm{d}, 1 \mathrm{H}, J=7.7 \mathrm{~Hz}), 6.59-6.76(\mathrm{~m}, 3 \mathrm{H}), 6.95-7.01(\mathrm{~m}, 2 \mathrm{H}), 7.32$ - $7.37(\mathrm{~m}, 2 \mathrm{H})$; HRMS (EI) $m / z[\mathrm{M}]^{+}$calcd. for $\mathrm{C}_{20} \mathrm{H}_{22} \mathrm{NO}_{2} \mathrm{~F} 327.1635$, found: 327.1628 .

Data for 31; colorless oil. $0.33 \mathrm{~g}, 98 \% .{ }^{1} \mathrm{H} \mathrm{NMR}\left(\mathrm{CDCl}_{3}, 300 \mathrm{MHz}\right) \delta 1.50-1.65$ $(\mathrm{m}, 4 \mathrm{H}), 2.15-2.28(\mathrm{~m}, 4 \mathrm{H}), 3.12(\mathrm{~d}, 2 \mathrm{H}, J=6.6 \mathrm{~Hz}), 3.85(\mathrm{~s}, 3 \mathrm{H}), 4.82-4.90(\mathrm{~m}$, $1 \mathrm{H}), 4.92-5.05(\mathrm{~m}, 1 \mathrm{H}), 6.37(\mathrm{dd}, 1 \mathrm{H}, J=5.9 \mathrm{~Hz}, 1.8 \mathrm{~Hz}), 6.57-6.84(\mathrm{~m}, 4 \mathrm{H})$, 7.18 - $7.39(\mathrm{~m}, 5 \mathrm{H})$; HRMS (EI) $\mathrm{m} / z[\mathrm{M}]^{+}$calcd. for $\mathrm{C}_{22} \mathrm{H}_{25} \mathrm{NO}_{2} 335.1885$, found: 335.1876 .

Data for 3m; colorless oil. $0.27 \mathrm{~g}, 78 \% .{ }^{1} \mathrm{H} \mathrm{NMR}\left(\mathrm{CDCl}_{3}, 300 \mathrm{MHz}\right) \delta 1.50-$ $1.65(\mathrm{~m}, 4 \mathrm{H}), 2.15-2.25(\mathrm{~m}, 4 \mathrm{H}), 2.30(\mathrm{~s}, 3 \mathrm{H}), 3.11(\mathrm{~d}, 2 \mathrm{H}, J=6.6 \mathrm{~Hz}), 3.85(\mathrm{~s}$, $3 \mathrm{H}), 4.82(\mathrm{dd}, 1 \mathrm{H}, J=6.6 \mathrm{~Hz}, 6.6 \mathrm{~Hz}), 6.40(\mathrm{~d}, 1 \mathrm{H}, J=6.2 \mathrm{~Hz}), 6.57-6.75(\mathrm{~m}$, $3 \mathrm{H}), 6.83-6.86(\mathrm{~m}, 1 \mathrm{H}), 7.10(\mathrm{~d}, 2 \mathrm{H}, J=8.0 \mathrm{~Hz}), 7.27(\mathrm{~d}, 2 \mathrm{H}, J=8.4 \mathrm{~Hz})$; HRMS (EI) $\mathrm{m} / z[\mathrm{M}]^{+}$calcd. for $\mathrm{C}_{23} \mathrm{H}_{27} \mathrm{NO}_{2} 349.2042$, found: 349.2044 .

Data for $3 \mathrm{n}$; colorless oil. $0.34 \mathrm{~g}, 95 \% .{ }^{1} \mathrm{H} \mathrm{NMR}\left(\mathrm{CDCl}_{3}, 300 \mathrm{MHz}\right) \delta 1.56-$ $1.59(\mathrm{~m}, 4 \mathrm{H}), 2.17-2.21(\mathrm{~m}, 4 \mathrm{H}), 3.11(\mathrm{dd}, 2 \mathrm{H}, J=5.9 \mathrm{~Hz}, 1.8 \mathrm{~Hz}), 3.86(\mathrm{~s}, 3 \mathrm{H})$, $4.80-4.87(\mathrm{~m}, 1 \mathrm{H}), 4.96$ (brs, $1 \mathrm{H}), 6.33(\mathrm{dd}, 1 \mathrm{H}, J=7.7 \mathrm{~Hz}, 1.5 \mathrm{~Hz}), 6.59-6.77$ $(\mathrm{m}, 3 \mathrm{H}), 6.82-6.87(\mathrm{~m}, 1 \mathrm{H}), 6.93-7.02(\mathrm{~m}, 2 \mathrm{H}), 7.33$ - $7.37(\mathrm{~m}, 2 \mathrm{H})$; HRMS (EI) $\mathrm{m} / z[\mathrm{M}]^{+}$calcd. for $\mathrm{C}_{22} \mathrm{H}_{24} \mathrm{NO}_{2} \mathrm{~F} 353.1791$, found: 353.1797 .

Data for 3o; colorless oil. $0.24 \mathrm{~g}, 97 \% .{ }^{1} \mathrm{H} \mathrm{NMR}\left(\mathrm{CDCl}_{3}, 500 \mathrm{MHz}\right) \delta 1.85$ (s, $3 \mathrm{H}), 2.09$ (s, 3H), $2.92(\mathrm{dd}, 1 \mathrm{H}, J=15.4 \mathrm{~Hz}, 7.7 \mathrm{~Hz}), 2.97(\mathrm{dd}, 1 \mathrm{H}, J=15.4 \mathrm{~Hz}$, $7.7 \mathrm{~Hz}), 3.86(\mathrm{~s}, 3 \mathrm{H}), 5.14-5.18(\mathrm{~m}, 1 \mathrm{H}), 6.06-6.07(\mathrm{~m}, 1 \mathrm{H}), 6.40-6.45(\mathrm{~m}$, $1 \mathrm{H}), 6.58-6.65(\mathrm{~m}, 1 \mathrm{H}), 6.68-6.80(\mathrm{~m}, 2 \mathrm{H}), 7.00-7.08(\mathrm{~m}, 2 \mathrm{H}), 7.17-7.25(\mathrm{~m}$, $1 \mathrm{H}), 7.35-7.37(\mathrm{~m}, 1 \mathrm{H})$; HRMS (EI) $\mathrm{m} / z[\mathrm{M}]^{+}$calcd. for $\mathrm{C}_{20} \mathrm{H}_{22} \mathrm{NO}_{2} \mathrm{~F} 327.1635$, found: 327.1637 .

\section{Cyclopropanation of 30 using trimethyloxosulfonium iodide}

To a stirred solution of $30(0.243 \mathrm{~g}, 0.74 \mathrm{mmol})$ in DMSO $(1 \mathrm{~mL})$ was added trimethyloxosulfonium iodide $(0.22 \mathrm{~g}, 1.0 \mathrm{mmol})$ and $\mathrm{NaH}(0.024 \mathrm{~g}, 1.0 \mathrm{mmol})$ at r.t. The mixture was stirred for 1 day and quenched with ice-water $(20 \mathrm{~mL})$. The mixture was extracted with ether, washed twice with water, and the organic layers were dried by $\mathrm{Na}_{2} \mathrm{SO}_{4}$. The solvent was removed at reduced pressure to give the product 40 as a white solid $(0.21 \mathrm{~g}, 65 \%)$. 
Data for 4o; ${ }^{1} \mathrm{H} \mathrm{NMR}\left(\mathrm{CDCl}_{3}, 500 \mathrm{MHz}\right) \delta 0.71-0.72(\mathrm{~m}, 1 \mathrm{H}), 0.86(\mathrm{~s}, 3 \mathrm{H})$, $1.06(\mathrm{~s}, 3 \mathrm{H}), 1.15-1.16(\mathrm{~m}, 1 \mathrm{H}), 1.75-1.80(\mathrm{~m}, 1 \mathrm{H}), 3.06(\mathrm{dd}, 1 \mathrm{H}, J=16.1 \mathrm{~Hz}$, $5.7 \mathrm{~Hz}$ ), 3.09 (dd, $1 \mathrm{H}, J=16.1 \mathrm{~Hz}, 5.7 \mathrm{~Hz}), 3.77$ (s, 3H), 5.08 (brs, 1H), 5.16 (dd, $1 \mathrm{H}, J=6.3 \mathrm{~Hz}, 6.3 \mathrm{~Hz}), 6.40-6.42(\mathrm{~m}, 1 \mathrm{H}), 6.60-6.65(\mathrm{~m}, 1 \mathrm{H}), 6.71-6.74(\mathrm{~m}$, 2H), $7.01-7.05(\mathrm{~m}, 2 \mathrm{H}), 7.17-7.25(\mathrm{~m}, 1 \mathrm{H}), 7.36-7.37$ (m, 1H); HRMS (EI) $\mathrm{m} / z[\mathrm{M}]^{+}$calcd. for $\mathrm{C}_{21} \mathrm{H}_{24} \mathrm{NO}_{2} \mathrm{~F} 341.1791$, found: 341.1796 .

\section{Acknowledgements}

We acknowledge JEOL Ltd. and Nihon Waters K. K. for measurement of HRMS.

\section{Conflicts of Interest}

The authors declare no conflicts of interest regarding the publication of this paper.

\section{References}

[1] Saranya, S., Harry, N.A., Krishman, K.K. and Anilkumar, G. (2018) Developments and Perspectives in the Asymmetric Mannich Reaction. Asian Journal of Organic Chemistry, 7, 613-633. https://doi.org/10.1002/ajoc.201700679

[2] Bala, S., Sharma, N., Kajal, A., Kamboj, S. and Saini, V. (2014) Mannich Bases: An Important Pharmacophore in Present Scenario. International Journal of Medicinal Chemistry, 2014, Article ID: 191072. https://doi.org/10.1155/2014/191072

[3] Karimi, B., Enders, D. and Jafari, E. (2013) Recent Advances in Metal-Catalyzed Asymmetric Mannich Reactions. Synthesis, 45, 2769-2812.

https://doi.org/10.1055/s-0033-1339479

[4] Subramaniapillai, S.G. (2013) Mannich Reaction: A Versatile and Convenient Approach to Bioactive Skeletons. Journal of Chemical Science, 125, 467-482. https://doi.org/10.1007/s12039-013-0405-y

[5] Córdova, A. (2004) The Direct Catalytic Asymmetric Mannich Reaction. Accounts of Chemical Research, 37, 102-112. https://doi.org/10.1021/ar0302311

[6] Roman, G. (2015) Mannich Bases in Medicinal Chemistry and Drug Design. European Journal of Medicinal Chemistry, 89, 743-816. https://doi.org/10.1016/j.ejmech.2014.10.076

[7] Boger, D.L. and Weinreb, S.N. (2012) In Hetero-Diels-Alder Methodology in Organic Synthesis. Academic Press, Inc., London.

[8] Buonora, P., Olsen, J.-C. and Oh, T. (2001) Recent Developments in Imino DielsAlder Reactions. Tetrahedron, 57, 6099-6138. https://doi.org/10.1016/S0040-4020(01)00438-0

[9] Kumatabara, Y., Kaneko, S., Nakata, S., Shirakawa, S. and Maruoka, K. (2016) Hydrogen-Bonding Catalysis of Tetraalkylammonium Salts in an Aza-Diels-Alder Reaction. Chemistry: An Asian Journal, 11, 2126-2129. https://doi.org/10.1002/asia.201600781

[10] Shang, D., Xin, J., Liu, Y., Zhou, X., Liu, X. and Feng, X. (2008) Enantioselective Aza-Diels-Alder Reaction of Aldimine with "Danishefsky-Type Diene" Catalyzed by Chiral Scandium(III)- $N, N$-Dioxide Complexes. Journal of Organic Chemistry, 73, 630-637. https://doi.org/10.1021/jo7021263

[11] Itoh, J., Fuchibe, K. and Akiyama, T. (2006) Chiral Brönsted Acid Catalyzed Enan- 
tioselective Aza-Diels-Alder Reaction of Brassard's Doeme with Imines. Angewandte Chemie International Edition, 45, 4796-4798. https://doi.org/10.1002/anie.200601345

[12] Sundén, H., Ibrahem, I., Eriksson, L. and Córdova, A. (2005) Direct Catalytic Enantioselective Aza-Diels-Alder Reactions. Angewandte Chemie International Edition, 44, 4877-4880. https://doi.org/10.1002/anie.200500811

[13] Loncaric, C., Manabe, K. and Kobayashi, S. (2003) AgOTf-Catalyzed Aza-Diels-Alder Reaction of Danishefsky's Diene with Imines in Water. Advanced Synthesis and Catalysis, 345, 475-477. https://doi.org/10.1002/adsc.200390052

[14] Mancheño, O.G., Arrayás, R.G. and Carretero, J.C. (2004) Chiral Copper Complexes of Phosphino Sulfenyl Ferrocenes as Efficient Catalysts for Enantioselective Formal Aza Diels-Alder Reaction of $N$-Sulfonyl Imines. Journal of American Chemical Society, 126, 456-457. https://doi.org/10.1021/ja038494y

[15] Kobayashi, S., Kusakabe, K. and Ishitani, H. (2000) Chiral Catalyst Optimization Using Both Solid-Phase and Liquid-Phase Methods in Asymmetric Aza Diels-Alder Reactions. Organic Letters, 2, 1225-1227. https://doi.org/10.1021/ol005656b

[16] Girling, P.R., Kiyoi, T. and Whiting, A. (2011) Mannich-Michael versus Formal Aza-Diels-Alder Approaches to Piperidone Derivatives. Organic and Biomolecular Chemistry, 9, 3105-3121. https://doi.org/10.1039/c0ob00996b

[17] Waldmann, H. and Braun, M. (1992) Asymmetric Tandem Mannich Reactions of Amino Acid Ester Imines with Danishefsky's Diene. Journal of Organic Chemistry, 57, 4444-4451. https://doi.org/10.1021/jo00042a027

[18] Kunz, H. and Pfrengle, W. (1989) Carbohydrates as Chiral Templates: Stereoselective Tandem Mannich-Michael Reactions for the Synthesis of Piperidine Alkaloids. Angewandte Chemie International Edition, 28, 1067-1068. https://doi.org/10.1002/anie.198910671

[19] Ishimaru, K. and Kojima, T. (2000) A Novel Approach for Mannich-Type Bases Having a Terminal Olefin: Zinc Triflate and Water-Promoted Cyclization/C-N Bond Cleavage Process. Journal of Organic Chemistry, 65, 8395-8398. https://doi.org/10.1021/jo0011888

[20] Ishimaru, K. and Kojima, T. (2003) Stereoselective Mannih-Type Reaction of Chiral Aldimines with 2-Silyloxybutadienes by Using Trifluoromethanesulfonic Acid. Tetrahedron Letters, 44, 5441-5444. https://doi.org/10.1016/S0040-4039(03)01314-5

[21] Ishimaru, K. and Kojima, T. (2003) Addition of Water to Zinc Triflate Promotes a Novel Reaction: Stereoselective Mannich-Type Reaction of Chiral Aldimines with 2-Silyloxybutadiene. Journal of Organic Chemistry, 68, 4959-4962. https://doi.org/10.1021/jo0300338

[22] Ishimaru, K. and Kojima, T. (2001) Stereoselective Synthesis of Mannich-Type Products Having a Terminal Olefin by Use of Benzaldiminetricarbonylchromium Derivatives. Tetrahedron Letters, 42, 5037-5039. https://doi.org/10.1016/S0040-4039(01)00912-1

[23] Adamo, I., Benedetti, F., Berti, F. and Campaner, P. (2006) Stereoselective Hydroazidation of Amino Enones: Synthesis of the Ritonavir/Lopinavir Core. Organic Letters, 8, 51-54. https://doi.org/10.1021/ol0524104

[24] Edwards, M.L., Ritter, H.W., Stemerick, D.M. and Stewart, K.T. (1983) Mannich Bases of 4-phenyl-3-buten-2-one. A New Class of Antiherpes Agent. Journal of Medicinal Chemistry, 26, 431-436. https://doi.org/10.1021/jm00357a020

[25] Dimmock, J.R., Nyathi, C.B. and Smith, P.J. (1979) Synthesis and Bioactivities of 
1-(hydroxyphenyl)-I-nonen-3-ones and Related Ethers and Esters. Journal of Pharmaceutical Science, 68, 1216-1221. https://doi.org/10.1002/jps.2600681006

[26] Dimmock, J.R., Nyathi, C.B. and Smith, P.J. (1978) Synthesis and Evaluation of 1-(hydroxyphenyl)-I-nonen-3-ones and Related Compounds for Antineoplastic and Antimicrobial Activities. Journal of Pharmaceutical Science, 67, 1543-1546. https://doi.org/10.1002/jps.2600671113

[27] Roselló, M.S., Pozo, C. and Fustero, S. (2016) A Decade of Advance in the Asymmetric Vinylogous Mannich Reaction. Synthesis, 48, 2553-2571. https://doi.org/10.1055/s-0035-1561650

[28] Lindemann, C. and Schneider, C. (2016) Quinolizine-Based Alkaloids: A General Catalytic, Highly Enantio and Diastereoselective Synthetic Approach. Synthesis, 48, 828-844. https://doi.org/10.1055/s-0035-1561289

[29] Ye, J.-L., Zhang, Y.-F., Liu, Y., Zhang, J.-Y., Ruan, Y.-P. and Huang, P.-Q. (2015) Studies on the Asymmetric Synthesis of Pandamarliactonines: An Unexpected syn-Selective Vinylogous Mannich Reaction of $\mathrm{N}$-tert-cutanesulfimines. Organic Chemistry Frontiers, 2, 697-704. https://doi.org/10.1039/C5QO00098J

[30] Wang, Q., Gemmeren, M. and List, B. (2014) Asymmetric Disulfonimide-Catalyzed Synthesis of d-amino-b-ketoester Derivatives by Vinylogous Mukaiyama-Mannich Reactions. Angewandte Chemie International Edition, 53, 13592-13595. https://doi.org/10.1002/anie.201407532

[31] Liu, L.-J. and Liu, J.-T. (2014) 2-Chlorotetrafluoroethanesulfinamide Induced Asymmetric Vinylogous Mannich Reaction. Tetrahedron, 70, 1236-1245.

https://doi.org/10.1016/j.tet.2013.12.071

[32] Guo, Y., Zhang, Y., Qi, L., Tian, F. and Wang, L. (2014) Organocatalytic Direct Asymmetric Vinylogous Mannich Reactions of g-butenolides with Isatin-Derived Ketimines. RSC Advances, 4, 27286-27289. https://doi.org/10.1039/c4ra04824e

[33] Sickert, M., Abels, F., Lang, M., Sieler, J., Birkemeyer, C. and Schneider, C. (2010) Brönsted Acid Catalyzed, Enantioselective Vinylogous Mannich Reaction. Chemistry: An European Journal, 16, 2806-2818. https://doi.org/10.1002/chem.200902537

[34] Gu, C.-L., Liu, L., Wang, D. and Chen, Y.-J. (2009) Tunable and Highly Regio- and Diastereoselective Vinylogous Mannich-Type Reaction of Dioxinone-Derived Silyl Dienolate. Journal of Organic Chemistry, 74, 5754-5757. https://doi.org/10.1021/jo900977y

[35] Sickert, M. and Schneider, C. (2008) The Enantioselective, Brönsted Acid Catalyzed, Vinylogous Mannich Reaction. Angewandte Chemie International Edition, 47, 3631-3634. https://doi.org/10.1002/anie.200800103

[36] Martin, S.F. (2002) Evolution of the Vinylogous Mannich Reaction as a Key Construction for Alkaloid Synthesis. Accounts of Chemical Research, 35, 895-904.

[37] Kawęcki, R. (2006) Aza Diels-Alder Reactions of Sulfinimines with the Rawal Diene. Tetrahedron: Asymmetry, 17, 1420-1423. https://doi.org/10.1016/j.tetasy.2006.04.026

[38] Xiong, Y., Mei, H., Han, J., Li, G. and Pan, Y. (2014) Asymmetric Synthesis of $\beta$-Amino- $\alpha$, $\beta$-Enones via Addition of $\alpha, \beta$-Unsaturated Ketone-Derived Enolates to Chiral $N$-Phosphonyl Imines. Tetrahedron Letters, 55, 2476-2479. https://doi.org/10.1016/j.tetlet.2014.03.005

[39] Reddy, A.A., Reddy, P.O. and Prasad, K.R. (2016) Synthesis of $\beta$-Amino-Substituted Enones by Addition of Substituted Methyl Enones to Sulfinimines: Application to the Total Synthesis of Alkaloids (+)-Lasubine II and (+)-241D and the Formal Total 
Synthesis of (-)-Lasubine I. Journal of Organic Chemistry, 81, 11363-11371. https://doi.org/10.1021/acs.joc.6b01541

[40] Yuan, Y., Li, X. and Ding, K. (2002) Acid-Free Aza Diels-Alder Reaction of Danishefsky's Diene with Imines. Organic Letters, 4, 3309-3311. https://doi.org/10.1021/ol0265822

[41] Pan, X., Huang, R., Zhang, J., Ding, L., Li, W., Zhang, Q. and Liu, F. (2012) Efficient Synthesis of Prasugrel, a Novel $\mathrm{P} 2_{\mathrm{Y} 12}$ Receptor Inhibitor. Tetrahedron Letters, 53, 5364-5366. https://doi.org/10.1016/j.tetlet.2012.07.071

[42] Jakubowski, J.A., Winters, K.J., Naganuma, H. and Wallentin, L. (2007) Prasugrel: A Novel Thienopyridine Antiplatelet Agent. A Review of Preclinical and Clinical Studies and the Mechanistic Bases for Its Distinct Antiplatelet Profile. Cardiovascular Drug Reviews, 25, 357-374. https://doi.org/10.1111/j.1527-3466.2007.00027.x 